\title{
Surgical management of anomalous origin of coronary artery from pulmonary artery
}

\author{
Amit Mishra $^{1}$
}

Received: 14 November 2019 / Revised: 5 January 2021 / Accepted: 12 January 2021 /Published online: 28 January 2021

(C) Indian Association of Cardiovascular-Thoracic Surgeons 2021

\begin{abstract}
Background Anomalous origin of coronary artery from pulmonary artery (AOCAPA), as is evident from the name, is defined as abnormal origin of either coronary artery from the pulmonary artery. The consequences vary in most cases and these anomalies lead to severe coronary hypo-perfusion and ventricular dysfunction. The common variants of this cardiac malformation are an anomalous origin of a left coronary artery from a pulmonary artery (ALCAPA) and anomalous origin of the right coronary artery from a pulmonary artery (ARCAPA). Another rare variant is left main coronary artery atresia that resembles ALCAPA in its mode of presentation. This article presents a single surgeon experience of managing this complex subset of the coronary anomaly from April 2006 to July 2019.

Material and methods The 105 patients, who underwent surgery for AOCAPA from April 2006 to July 2019, have been included in the study. The patients have been analysed by follow-up echocardiography and electrocardiography (ECG) at our hospital by paediatric cardiologists. Out of 105 patients of AOCAPA, 98 (93.3\%) patients underwent ALCAPA repair, of which $59(60.2 \%)$ were males and $39(39.7 \%)$ were females. Four out of five patients, who had an anomalous origin of the left coronary artery from the right pulmonary artery (ALCARPA), had an intramural aortic course. Three patients ( $3 \%$ ) had left main coronary artery atresia and four patients (4\%) had ARCAPA. It may be mentioned that seven infants (7.14\%) and one adult patient (1\%) underwent concomitant mitral valve repair. All the patients with ALCAPA, left main coronary artery atresia and ARCAPA, and 1 of the patients with ALCARPA, underwent coronary relocation. In four out of five patients with ALCARPA, unroofing of intraaortic intramural course was performed.

Results Out of 105 patients of AOCAPA, 9 (8.5\%) patients had in-hospital mortality. Five infants (5.0\%) with ALCAPA and one patient $(1 \%)$ with ALCARPA died in the post-operative period due to severe left ventricular dysfunction, mitral regurgitation (MR) and sepsis. One adult patient (1\%) with ALCAPA, who underwent coronary relocation using in situ trap door technique and mitral valve (MV) repair, died due to massive intracranial bleeding. Two patients out of three $(66.6 \%)$ with left main coronary atresia died in intensive care unit (ICU) after 3rd and 4th postoperative day, due to low cardiac output, severe ventricular dysfunction and severe MR. Patients were followed up for a median 5.9 years. Seven patients were lost to follow-up, including the sole survivor of left main coronary atresia, after a median follow-up of 4 years after surgery. Three patients underwent mitral valve replacement for progressive residual MR. There has been no late mortality.

Conclusion AOCAPA is a rare congenital cardiac anomaly, which usually presents in infancy with left ventricular dysfunction and mitral valve regurgitation. Early diagnosis and surgical re-establishment of the dual coronary system has given gratifying results, with improvement in left ventricular function in survivors. Mitral valve intervention for MR was required, in both early and late phases.
\end{abstract}

Keywords AOCAPA: Anomalous origin of coronary artery from pulmonary artery - ALCAPA: Anomalous origin of left coronary artery from pulmonary artery - ALCARPA: Anomalous origin of left coronary artery from right pulmonary artery · ARCAPA: Anomalous origin of right coronary artery from pulmonary artery $\cdot$ Left main atresia $\cdot$ Congenital coronary anomaly

Amit Mishra

drmishraamit@gmail.com

1 Department of Cardio vascular and Thoracic Surgery, U. N. Mehta Institute of Cardiology and Research Center (affiliated to BJ medical college, Ahmedabad), New Civil Hospital Campus, Asarwa, Ahmedabad, Gujarat 380016, India

\section{Introduction}

Anomalous origin of the coronary artery from the pulmonary artery (AOCAPA) is a rare congenital cardiac anomaly, with an incidence of $0.01 \%$ [1] in the general population. Anomalous 
origin of the left coronary artery from the pulmonary artery (ALCAPA) is the commonest, accounting for 1 in $3,00,000$ live births [2]. It can easily be diagnosed in infancy, as the patient shows clear-cut symptoms. The problem calls for timely treatment, as almost $90 \%$ of patients succumb to the ailment in the first year itself $[2,3]$. However, a fraction of patients with ALCAPA may reach the late adult stage, if either the symptoms are dormant, or they may present with symptomatic myocardial infraction (MI) or as severe mitral regurgitation (MR), due to chronic ischemia. Anomalous origin of the left coronary artery from the junction of the main and the right pulmonary artery (ALCARPA) is also a rare form of ALCAPA. In this, the left coronary artery arises from the junction of the main and right pulmonary artery (PA) and enters into the aorta with an intramural or extramural (Fig. 1) course in the aortic wall. It exits through the normal course of the left main coronary artery [4]. Left main coronary artery atresia is also an uncommon anomaly, in which there is no left coronary ostia and the proximal left main coronary trunk ends blindly. The blood flows from the right coronary artery (RCA) to the left, through small collateral arteries in retrograde manner, in at least one of the left side arteries. Its clinical presentation is similar to ALCAPA with extensive collaterals in the right ventricle and area around the main PA [5]. Anomalous origin of the RCA from the pulmonary artery (ARCAPA) accounts for $0.002 \%$ of cases with congenital heart disease (CHD) [6]. It is a relatively benign condition and presents usually in adolescence and adults. Almost $60 \%$ of ARCAPA patients are asymptomatic and have the best prognosis amongst all forms of AOCAPA [6, 7].

\section{Material and methods}

A retrospective case records review was made of all patients with diagnosis of AOCAPA. During the study

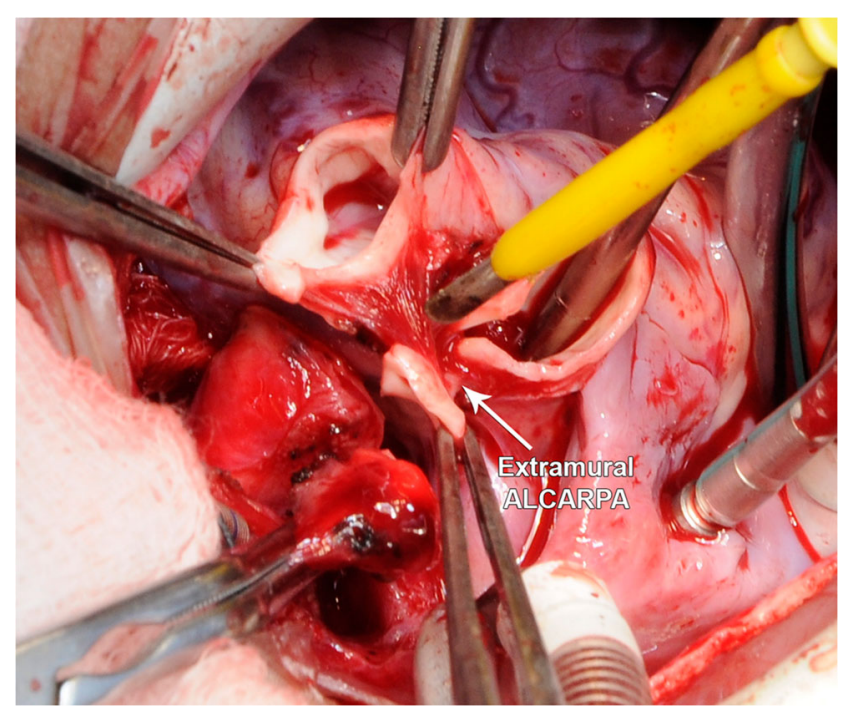

Fig. 1 Showing extramural ARCAPA period, April 2006 to July 2019, 105 patients underwent surgery for AOCAPA, 98 patients (93\%) had ALCAPA, including 5 patients $(4.7 \%)$ with ALCARPA; 3 patients (3\%) had left main coronary artery atresia and 4 patients (4\%) had ARCAPA. The median age at presentation was 5.8 months (range 14 days to 45 years). There were two adult patients (2\%). Fifty-nine were males $(60 \%)$ and 39 $(40 \%)$ were females. Three $(3 \%)$ had left coronary artery (LCA) arising from non-facing sinus (Fig. 2). Twenty-five $(25.5 \%)$ of the $33(33.6 \%)$ patients underwent surgery in early infancy ( $<6$ months), and $51(52 \%)$ patients in late infancy ( $>6$ months). Seven patients $(6.8 \%)$ out of 98 with ALCAPA had associated lesions. Two patients (2\%) had secundum atrial septal defect (ASD), while one (1\%) had primum ASD with a double orifice mitral valve (MV). This patient underwent MV repair and closure of the ostium primum ASD. One patient (1\%) had large patent ductus arteriosus (PDA) with severe pulmonary arterial hypertension (PAH), and one 3-month-old child had a large aortapulmonary (AP) window with left coronary artery arising from the PA side of the window (Fig. 3). He had good left ventricular (LV) function with mild MR. One patient (1\%) had a large ventricular septal defect (VSD) with severe PAH. One (1\%) of the patients with ALCAPA had accessory left anterior descending (LAD) coronary artery from RCA (Fig. 4), and he had mild MR and moderate LV dysfunction. Seven infants $(6.6 \%)$ and two (2\%) adults ALCAPA patients had severe MR needing MV repair or replacement. Three patients $(2.94 \%)$ had left main coronary artery atresia and 4 patients (3.8\%) had ARCAPA (one of them had an intramural course in PA with moderate size secundum ASD).

12-lead electrocardiography (ECG) and transthoracic echocardiographic examination was performed on all patients. Ejection fraction (EF) and fractional shorting (FS) were employed to classify LV systolic dysfunction. According to this, LV systolic function was accepted as normal when LVEF was $\geq 60 \%$, and there was mild, moderate and severe systolic dysfunction if LVEF was 50-59\%, 35-49\% and less than $35 \%$, respectively. Mitral regurgitation was graded as I (none/trivial), II (mild), III (moderate) and IV (severe). Coronary angiography and computed tomography (CT) pulmonary angiogram were performed in selected patients. All patients with ALCAPA, ARCAPA, and 2 patients with left main coronary artery atresia underwent left main coronary artery transfer to the aorta. Four patients $(3.8 \%)$ with ALCARPA underwent unroofing of an intramural intraaortic course and one patient with extramural course underwent coronary relocation (Fig. 1). None of the ALCAPA patients were put on extracorporeal membrane oxygenation (ECMO) support in the immediate post-operative period. The data on follow-up, with last clinical, ECG and echocardiography evaluation, and any further surgical intervention, were noted. 
Fig. 2 Showing ALCAPA origin from non-facing sinus

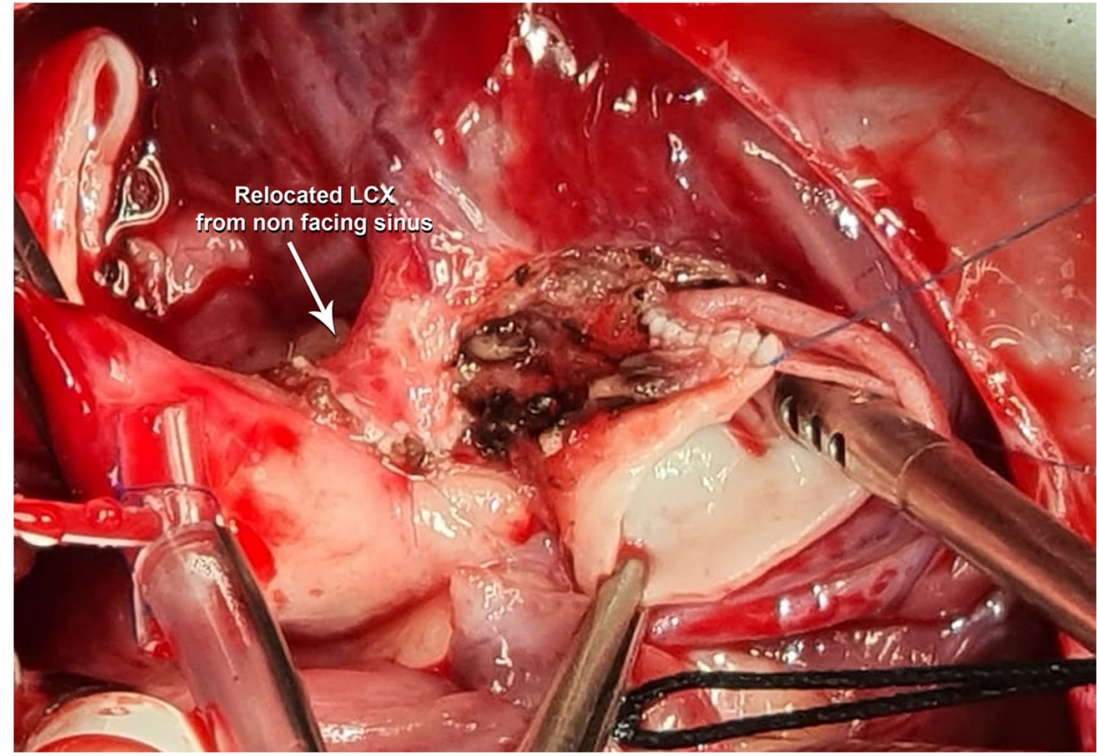

\section{Surgical technique for ALCAPA (Fig. 5a-e)}

Diagnosis was the indication for surgery. Patients in sepsis were stabilised preoperatively. Chest was opened through midline sternotomy. Pericardium was opened. Collaterals between aorta and PA groove were cauterised. Branch PAs were dissected and looped, PDA was dissected. Using standard technique of caval cannulation, PDA/ligamentum arteriosus was ligated and divided. Cardioplegic arrest was achieved using ante-grade cardioplegia via aortic root, after occlusion of branch PAs. Left atrial (LA) vent was placed through patent foramen ovale (PFO). Main PA was incised just below the level of right PA. After division of anterior half of the main PA, position of the coronary artery ostium was confirmed (just to avoid any injury to coronary artery in case of misdiagnosed

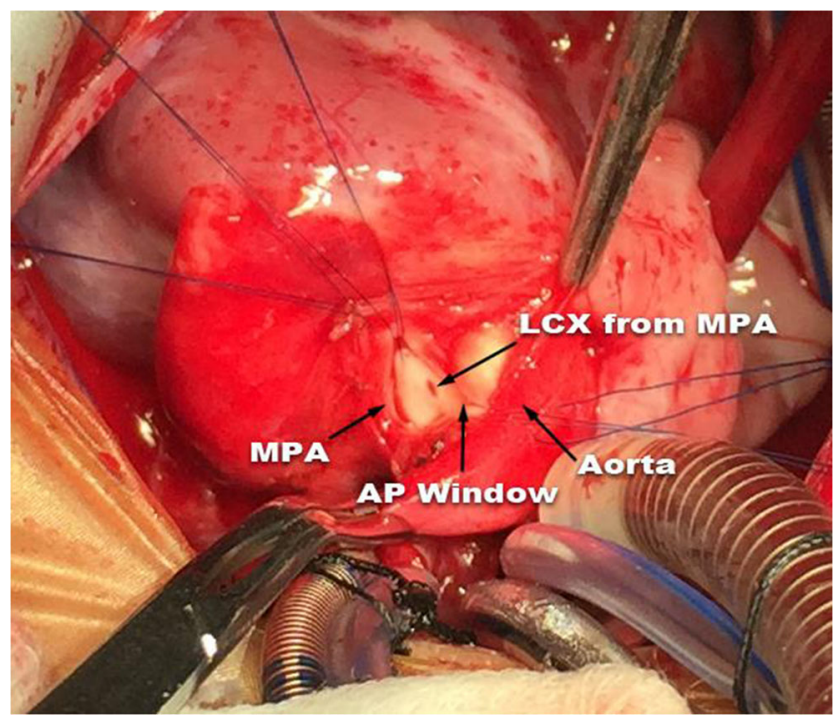

Fig. 3 Operative photographs showing AP window with ALCAPA
ALCARPA). Main PA was transected, position of the anomalous artery was confirmed and coronary button was excised for transfer to aorta (Fig. 5a). Coronary button was dissected off the epicardium for a centimetre to avoid any unnecessary traction on the coronary button artery. Collateral branches, which were divided during dissection of the coronary button, were cauterised or sutured with 7-0 polypropylene. Defect in the main PA was repaired with autologous glutaraldehydetreated pericardium (Fig. 5b). A stay stitch (Fig. 5b) was taken at the expected site of transfer, at postero-lateral aspect of aorta at nearly 7 o'clock position, which is usually slightly difficult for the surgeon to see. Stay suture was pulled towards the first assistant and the aorta was cut opened at the site of stay suture

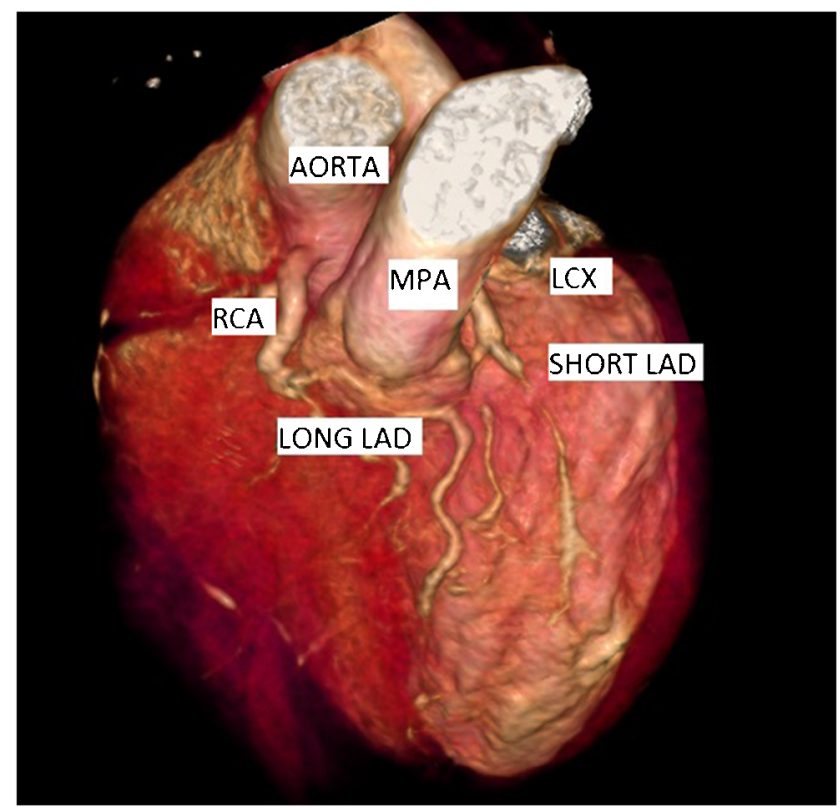

Fig. 4 CT coronary angiography demonstrating anomalous origin of LAD with combination of dual LAD and ALCAPA 

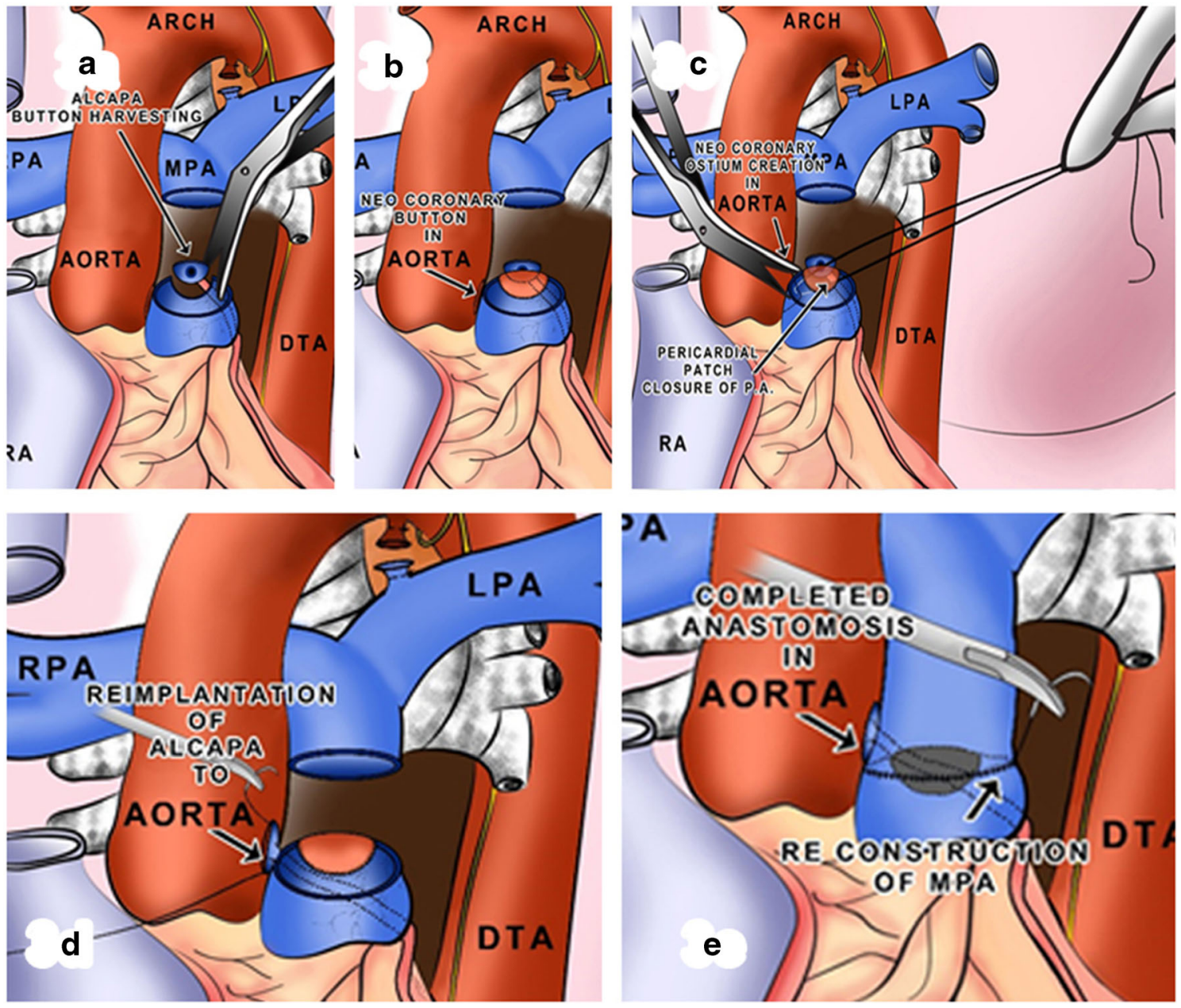

Fig. 5 Schematic diagrams showing surgical technique for ALCAPA. a The main pulmonary artery transected above the origin of LCA and coronary button being harvested. b Pericardial patch reconstruction of MPA and creation of aortotomy at postero-lateral aspect of aorta. c

(Fig. 5b, c). The opening was inspected and was further enlarged using a punch. The coronary artery was transferred using 7-0 polypropylene continuous suture (Fig. $5 \mathrm{~d}$ ). We never had any injury to aortic valve leaflet or the commissure. Although we have only two adult ALCAPA in our series, we believe that the direct transfer of coronary artery is possible in adult patients using in situ lateral or medial base trap door technique or by using native tissue for coronary artery reconstruction [8]. Small dose of cardioplegia was given to check for any bleeding from anastomotic site or from any collateral vessel. We usually do MV repair only if there is some organic cause for MR, like cleft or prolapse, or in cases of moderate to severe MR, using posterior suture annuloplasty. The other associated lesions are managed after coronary transfer (MV repair, VSD, ASD closure). In one of our patients, who had a large AP window (Fig. 3), the patch between the aorta and PA was placed in such a way, as to leave the left coronary artery towards aortic side of the patch. Pulmonary artery posterior layer was constructed on clamp and remaining anterior part
Opening for transfer of LCA at posterolateral aspect of aorta. d Reimplantaion of left coronary artery. e Reconstruction of main pulmonary artery

was reconstructed off-clamp (Fig. 5e). The bypass was weaned off using adrenaline, milrinone or levosimendan support. We usually do not place LA line as a routine and we measured LA pressures and performed transesophageal echocardiography (TEE) only when either MV repair was performed, or if there was difficulty in weaning from bypass. It was not uncommon to have borderline haemodynamics after coming off bypass which gradually improved with cardio pulmonary bypass (CPB) support. Occasionally, there was some bleeding from left postero-lateral aspect of main PA, which usually settled with protamine administration or glue application. We preferred to close the chest after surgery, and chest was only left open, to be closed later, if there was medical bleeding or borderline haemodynamics.

\section{Surgical technique for ALCARPA (Fig. 6a-e)}

If the diagnosis is known before surgery, the entry of the left coronary artery, from the junction of main PA or right PA to 
posterior surface of ascending aorta, can be seen (Fig. 6a, $\mathrm{b}, \mathrm{d})$. After giving cardioplegia from ascending aorta and main PA, after occlusion of branch PAs, the aorta was transected just at the level of right PA. It is difficult to understand the intramural intra-aortic course of left main coronary artery from inside the aorta, so it is safer to open the right PA and identify the opening of coronary artery from right PA and the entry point in aorta. The coronary artery can be disconnected from right PA and its opening can be unroofed using Potts scissors (Fig.6c), until the lower end of its intramural course, which is usually a bigger opening of the left main coronary artery. The unroofed edges are re-endothelialised (Fig. 6e). The junction of main PA and right PA can be reconstructed using pericardial patch and the aorta can be reconstructed. If the course of ALCARPA is extramural, then it has to be relocated.

Surgical technique for left main coronary artery atresia is demonstrated in Fig.7a-e. Left main coronary atresia should always be suspected first, if the anomalous LCA is not seen to be arising from the main PA during surgery and the left main coronary artery should be looked for behind the main PA. In our experience, it usually ran behind the main PA and ended blindly as a bulbous end (Fig. 7a), surrounded by extensive collaterals, just 3-4 mm short of postero-lateral aspect of the aorta from where the left main coronary artery arises (Fig. 7b). If the diagnosis was known before surgery, then after giving cardioplegia through aortic root, the main PA was dissected, as is done in ALCAPA. The bulbous portion of the left main coronary artery was identified and dissected off the epicardium for about a centimetre, and the blind end was cut opened (Fig. 7c, d). Usually, the cut end was slightly thickened and could easily be sutured to the aorta at 7 o'clock position, like proximal coronary anastomosis (Fig. 7e). Small dose of cardioplegia is given through the aortic root and flows through the coronary artery and any bleeding from collaterals was checked and fixed at the same time. Usually, there were a lot of collaterals around the left main coronary artery and special attention was required to control bleeding from collaterals. In our experience, patients with left main coronary atresia were

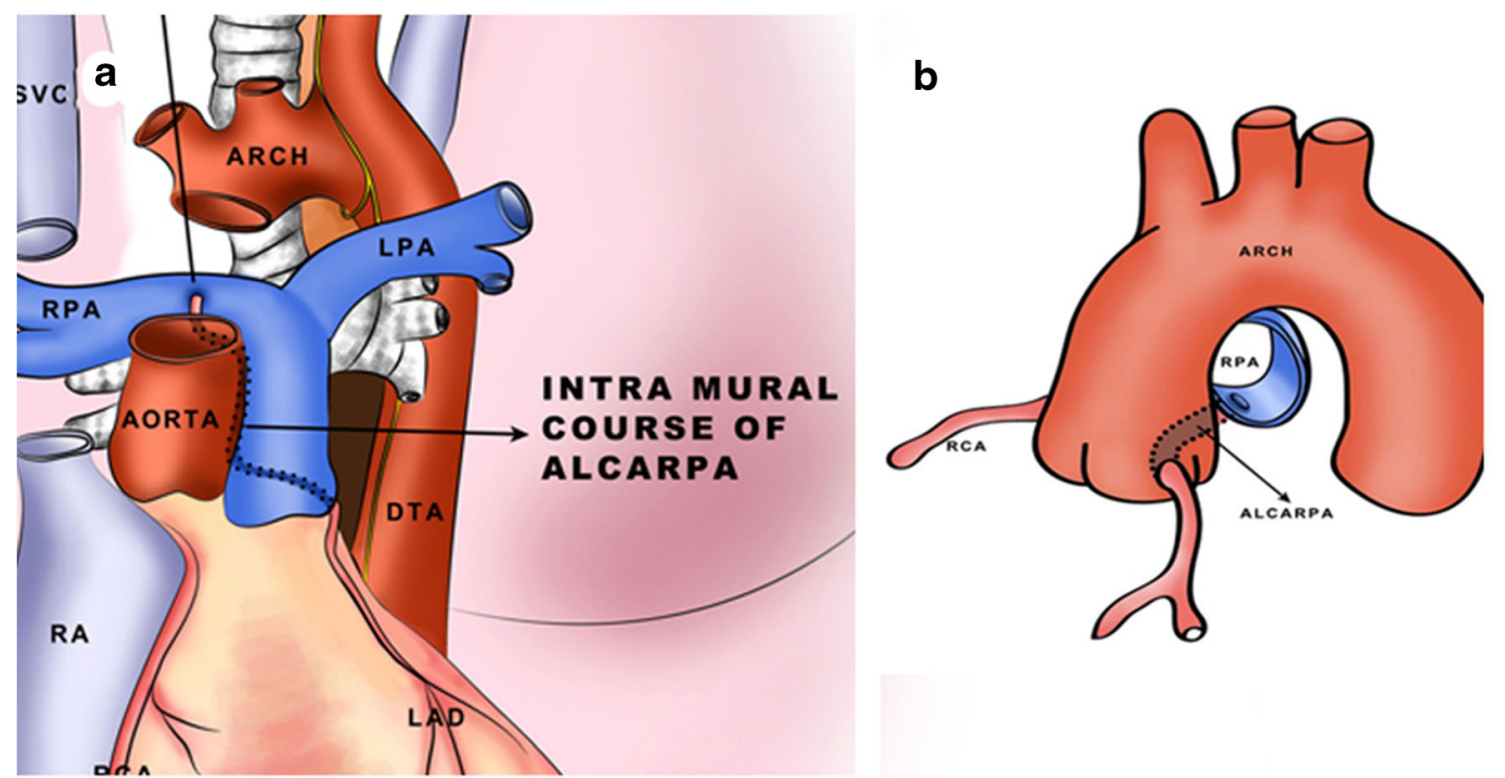

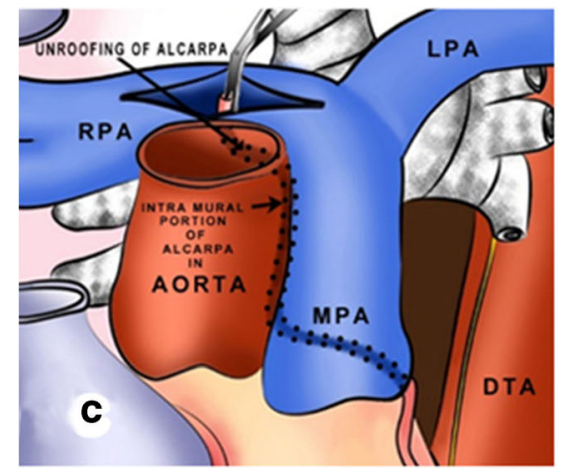
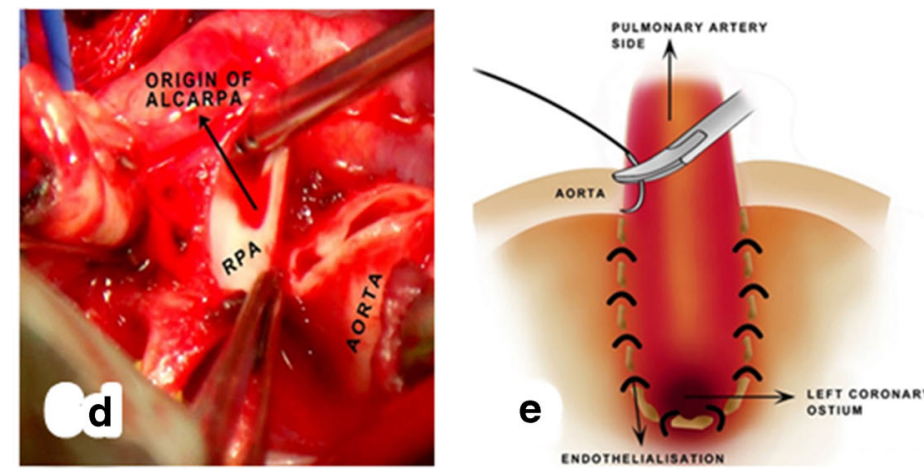

Fig. 6 Schematic figure showing technique for ALCARPA surgery. a Schematic cut section of aorta at level of RPA to expose origin of left coronary artery from right pulmonary artery. b Schematic view of anomalous origin of left coronary from RPA with vertical descending intramural course. c Schematic diagram showing unroofing of the intramural course of left coronary artery. d Right pulmonary artery opened to expose origin of anomalous left coronary artery from right pulmonary artery (ALCARPA). e Schematic view of endothelialisation 

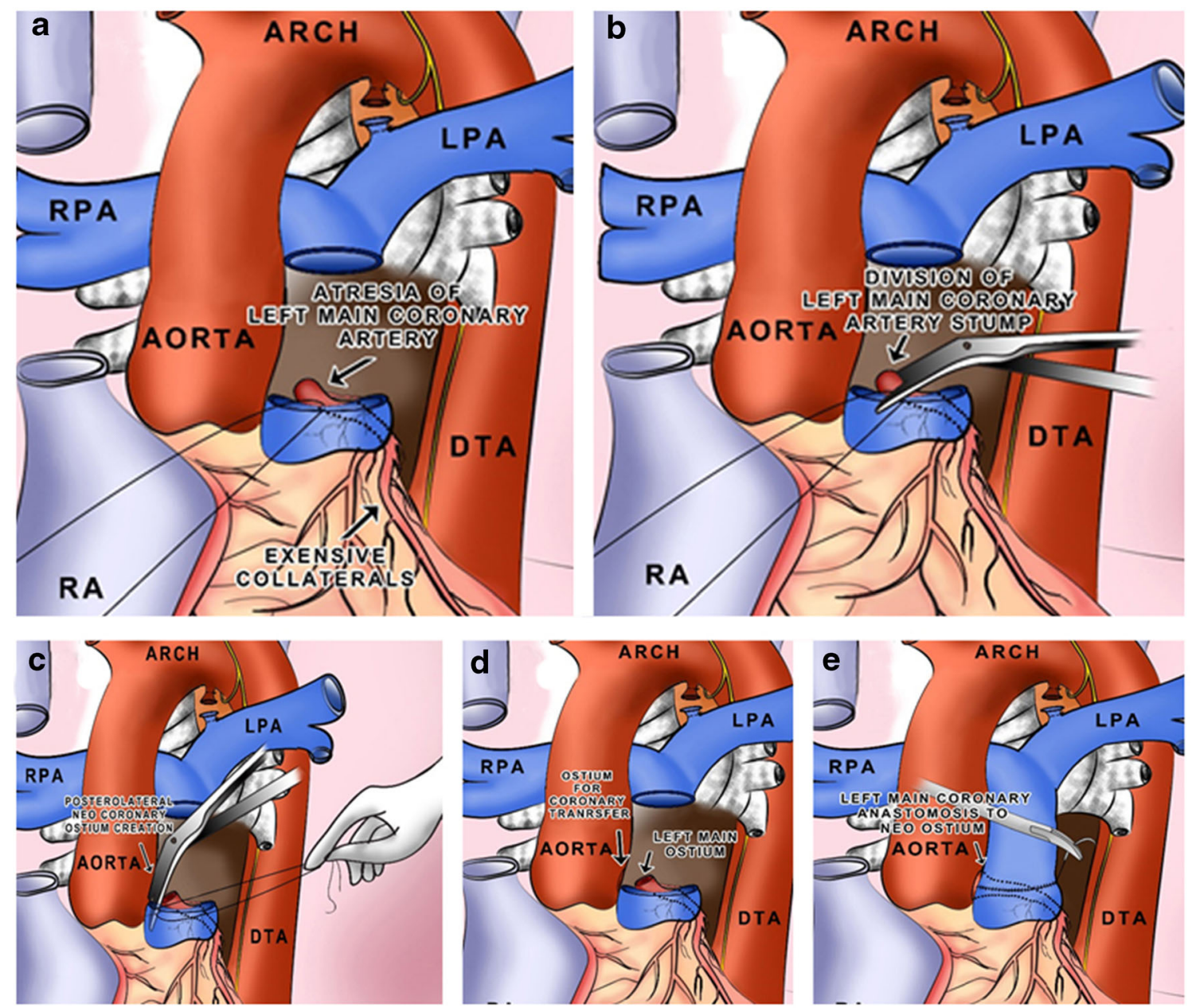

Fig. 7 Schematic diagrams showing surgical technique for left main atresia. a The main pulmonary artery transected, showing atretic left main coronary artery 3-4 mm from ascending aorta. b Main pulmonary artery retracted anteriorly and the distal end of left main coronary artery stump is cut. c Showing creation of opening for transfer of LCA at postero-lateral aspect of aorta. d Showing opening at postero-lateral aspect of aorta and cut end of left main coronary artery. e Showing transfer of left main coronary artery to aorta and pulmonary artery reconstruction associated with severe MR and we did posterior suture annuloplasty in both our patients. Main PA can be reconstructed as described in ALCAPA (Fig. 7e). These patients may need prolonged bypass support or may even need ECMO support (we did not have access to ECMO, when we operated on these patients).

\section{Surgical technique for ARCAPA (Fig. 8a-c)}

Standard CPB and cardioplegic arrest, by giving cardioplegia through both aorta and main PA (well above the origin of RCA), after occlusion of branch PAs, were utilised. RCA button was excised and the resulting defect was repaired using a glutaraldehyde-treated pericardial patch (Fig. 8a, b). The coronary artery was mobilised about a centimetre and was transferred using punch hole technique to ascending aorta (Fig. 8c). The associated lesions were treated at the same time and bypass was weaned off in a routine fashion. As the myocardium is well preserved, these patients behave very well in the post-operative period and can be weaned off ventilator within a few hours.

\section{Statistical analysis}

All the statistical analyses for the study were performed using SPSS software v 20.0 (Chicago, IL, USA). The continuous and normally distributed data was expressed as mean $\pm \mathrm{SD}$, whereas categorical data was presented as frequency and percentage. As age of the patients at the time of operation and follow-up duration of the patient population were heterogeneous and non-normally distributed, they were expressed as median and range. Survival analysis was also undertaken and is presented as Kaplan-Meier survival curve. The patients were grouped according to the age at the time of operation, and the improvement in LV function and MR during postoperative follow-up, according to the groups, was assessed and plotted. 


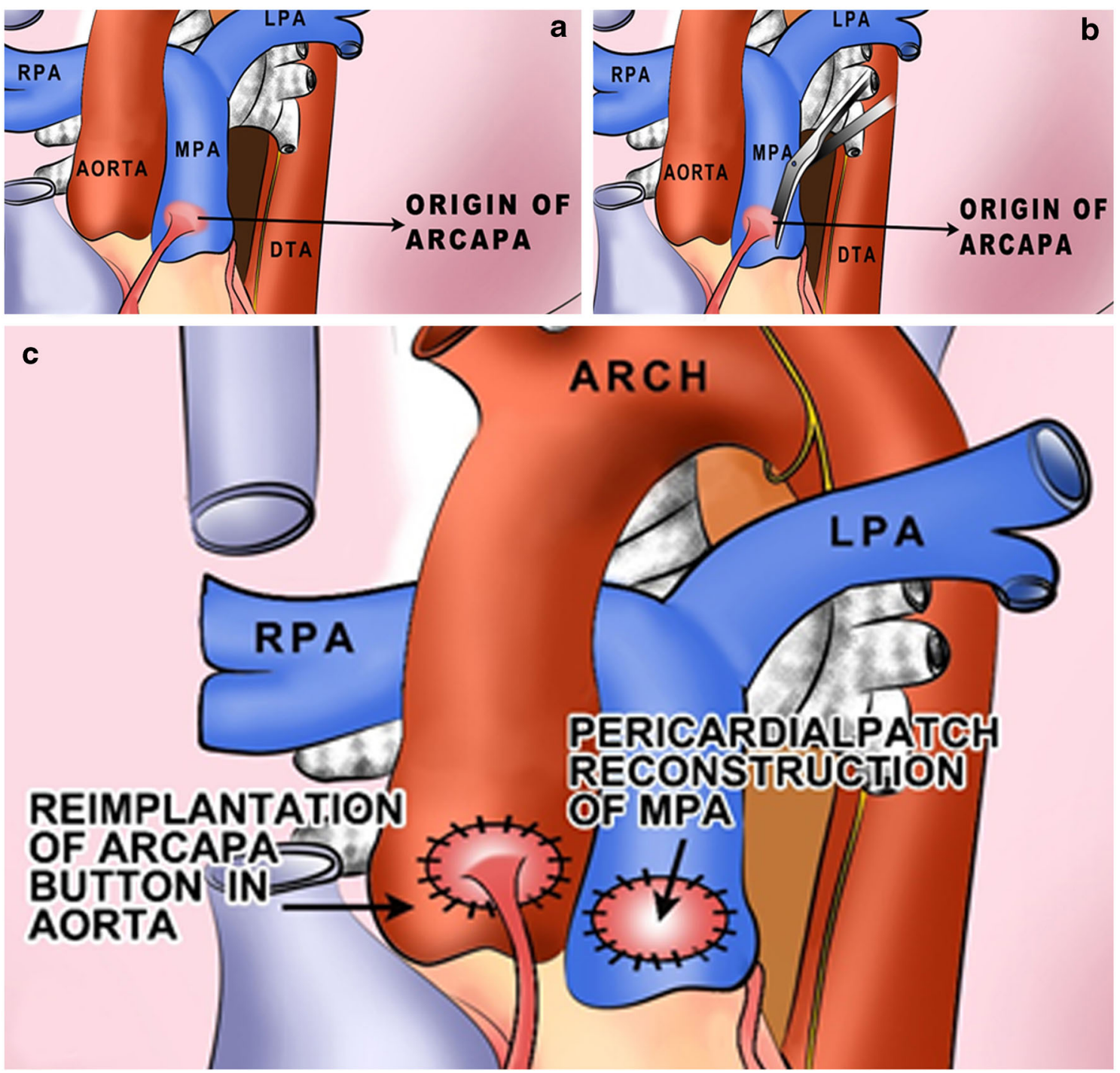

Fig. 8 Schematic diagrams showing surgical technique for ARCAPA. a Showing ARCAPA. b Showing RCA button harvesting. $\mathbf{c}$ Showing relocation of RCA and pericardial patch reconstruction of MPA

\section{Results}

\section{Patient characteristics}

Out of 105 patients of AOCAPA, 98 (93.3\%) patients underwent ALCAPA repair. Median age at operation was 5.8 months. Four (4\%) out of five patients had ALCARPA with intramural aortic course. Three patients $(3.06 \%)$ had left main coronary artery atresia and four (4\%) patients had ARCAPA. Seven infants (7.14\%) and one (1\%) adult patient underwent concomitant MV repair. One adult patient underwent MV replacement.

The patients, presenting in early infancy ( $<6$ months), had mean LVEF of $43.5 \%( \pm 5.1 \%)$ and those presented in late infancy ( $>6$ months) had mean LVEF of $38.6 \%( \pm 4.6 \%)$. Twenty-five $(25.5 \%)$ of the $33(33.6 \%)$ patients underwent surgery in early infancy ( $<6$ months), $25(25.5 \%)$ had grade II to III MR and $8(8.1 \%)$ patients had grade III to IV MR. Of the $51(52 \%)$ patients who underwent surgery in late infancy (>6 months), 38 (38.7\%) had grade III to IV MR and 13 (13.2\%) patients had grade IV MR.

Mean CPB and aortic cross-clamp time were $93 \pm 9$ min (81-102 $\mathrm{min}$ ), and $63 \pm 7 \mathrm{~min}$ (55-72 $\mathrm{min}$ ) respectively.

On follow-up, all patients underwent ECG and echocardiographic examination.

\section{Early mortality (Fig. 9)}

Early mortality was $8.5 \%$ (9 out of 105) in the entire cohort. Five $(4.7 \%)$ infants with ALCAPA and one $(0.95 \%)$ patient with ALCARPA died in post-operative period due to severe LV dysfunction, MR and arrhythmias. One $(0.95 \%)$ adult patient with ALCAPA died due to massive intracranial bleeding. Two $(1.9 \%)$ patients with left main coronary artery atresia died in intensive care unit (ICU), after 3rd and 4th post- 
Fig. 9 Survival analysis for ALCAPA patients using KaplanMeier curve

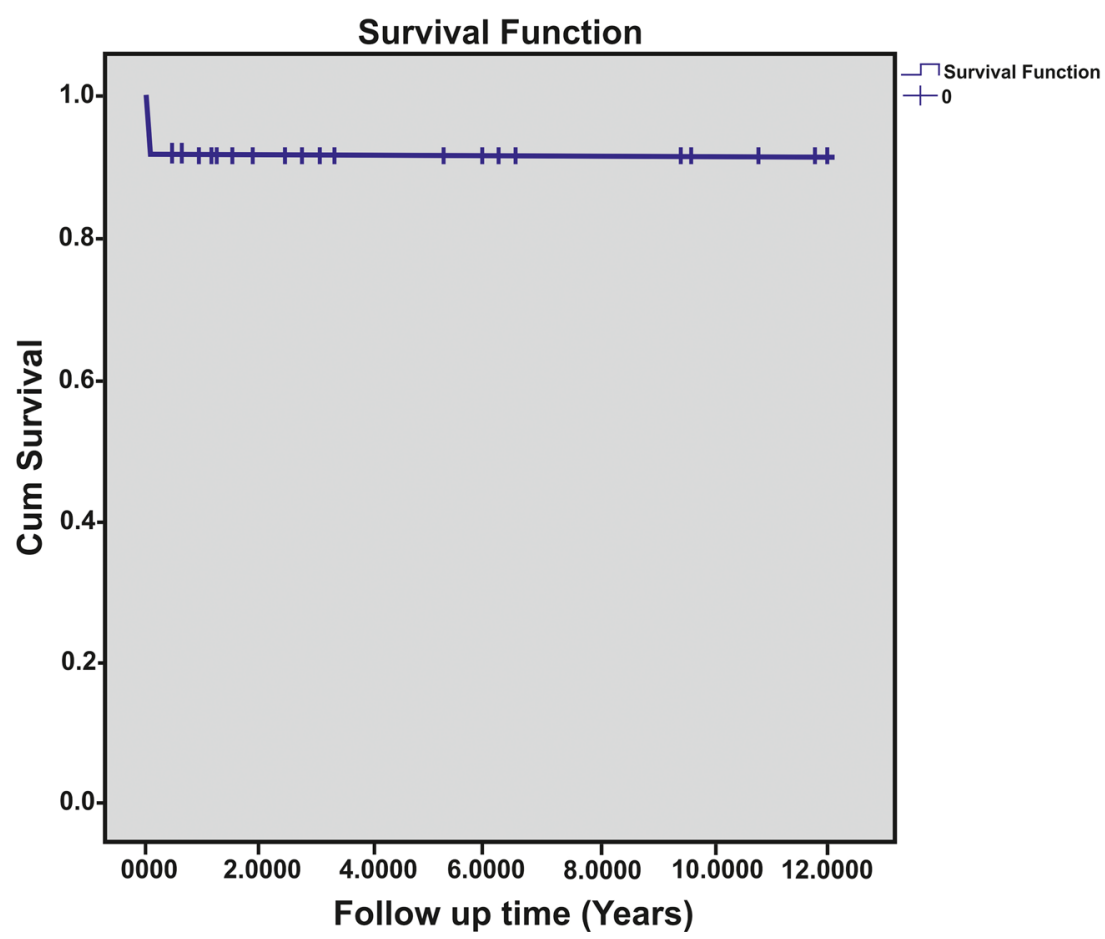

operative day, due to low cardiac output, severe ventricular dysfunction and severe MR.

\section{LV function (Fig. 10)}

Median follow-up of ALCAPA patients was 5.9 years. One new born, who had moderate LV dysfunction and mild MR, had normal LV function and no MR after 6 months of surgery. Thirty-three (33.6\%) patients, who underwent surgery in early infancy $(<6$ months), had good recovery of LV function (mean LVEF 50 to 55\%) and fractional shortening of 25 to $28 \%$. Fifty-one (52\%) patients, who underwent surgery in late infancy, had impairment of LV function (mean LVEF 40 to $50 \%$ and fractional shortening of 20 to 25 ) and 14 (14.2\%) operated after infancy, without any left to right shunt but with severe ventricular dysfunction, had persistent mild to moderate $\mathrm{LV}$ dysfunction with gradual improvement of $\mathrm{LV}$ function (mean LVEF between 40 and $45 \%$ and fractional shortening 18 to $21 \%$ ).

\section{Mitral regurgitation (Fig. 11)}

Thirty-three (33.6\%) patients, who underwent surgery in early infancy with moderate MR, continued to have mild regurgitation on follow-up. Fifty-one (52\%) patients operated after 6 months of age continue to have mild to moderate MR on follow-up. Fourteen (14.2\%) patients, who were operated after infancy, continue to have mild to moderate MR on follow-up and three of them underwent MV replacement after 3 years, for unrepaired progressive severe MR.
We have started repairing MVs in patients with severe MR presenting in late infancy, after July 2012. Seven patients (6.86\%) presented after infancy. They had severe MR before surgery and had undergone MV repair, but continued to have mild to moderate MR and were on medications.

\section{Presence of left to right shunt}

Six patients (5.7\%) with left to right shunt and one (0.9\%) with accessary LAD from RCA had normal preoperative LV function and no MR. This was no doubt secondary to severe pulmonary hypertension limiting the retrograde steal from the anomalous LCA.

\section{Follow-up}

Eighty-five patients were followed up until July 2019. Median follow-up was 5.9 years (1.5-12.5 years). Seven patients were lost to follow-up after a median follow-up of 4 years (3.26 years). Three patients underwent MV replacement for severe progressive unrepaired MR. All patients were in sinus rhythm, and no ischemia or arrhythmias were identified. None of our patients had aortic coronary stenosis. No late deaths were reported in any patient with AOCAPA, to date.

\section{Discussion}

Anomalous origin of the coronary artery from the PA is a rare congenital cardiac anomaly, with an incidence of $0.01 \%$ [1] in 


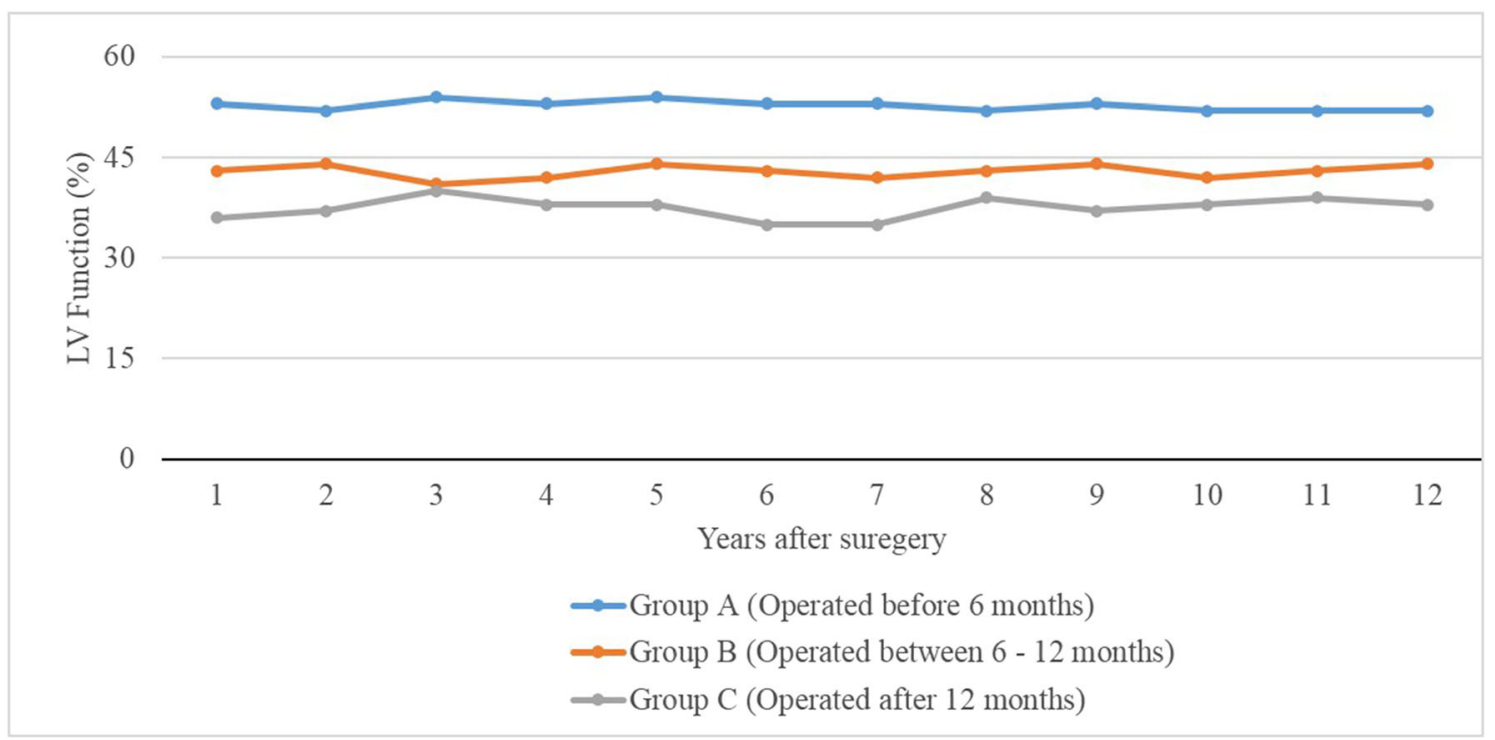

Fig. 10 Time course for left ventricular ejection fraction change in ALCAPA patients

the general population. ALCAPA is the commonest, accounting for 1 in 3,00,000 live births. It can easily be diagnosed in infancy as the patient shows clear-cut symptoms [2]. The problem calls for timely treatment, as almost $90 \%$ of patients succumb to the ailment in the first year itself $[2,3]$.

ALCAPA patients are usually infants, who after birth register decreased pulmonary vascular resistance causing left to right shunt [2]. We have operated only one 14-day-old baby, who had mild preoperative MR and moderate LV dysfunction with $\mathrm{EF}$ of $45 \%$. One of our patients, a 3-month-old infant, was diagnosed to have ALCAPA during surgery for the AP window. The patch was placed in such a manner that the LCA was placed on the aortic side of the patch. Only 11 cases of ALCAPA with AP window have been reported to date in English medical literature [9]. Several techniques for surgical management of ALCAPA have been reported since Sabiston and associates described ligation of the LCA at its origin [10]. Neches and colleagues [11] first time reported direct implantation of left coronary into the aorta, with a good result, and it is now universally accepted. We also believe that direct aortic implantation is the treatment of choice for ALCAPA in infants [12], even if the coronary artery is arising from non-facing sinus. Preservation of two coronary system helps in early recovery of LV function. In infants, it is possible to mobilise the
Fig. 11 Time course for MR in ALCAPA patients

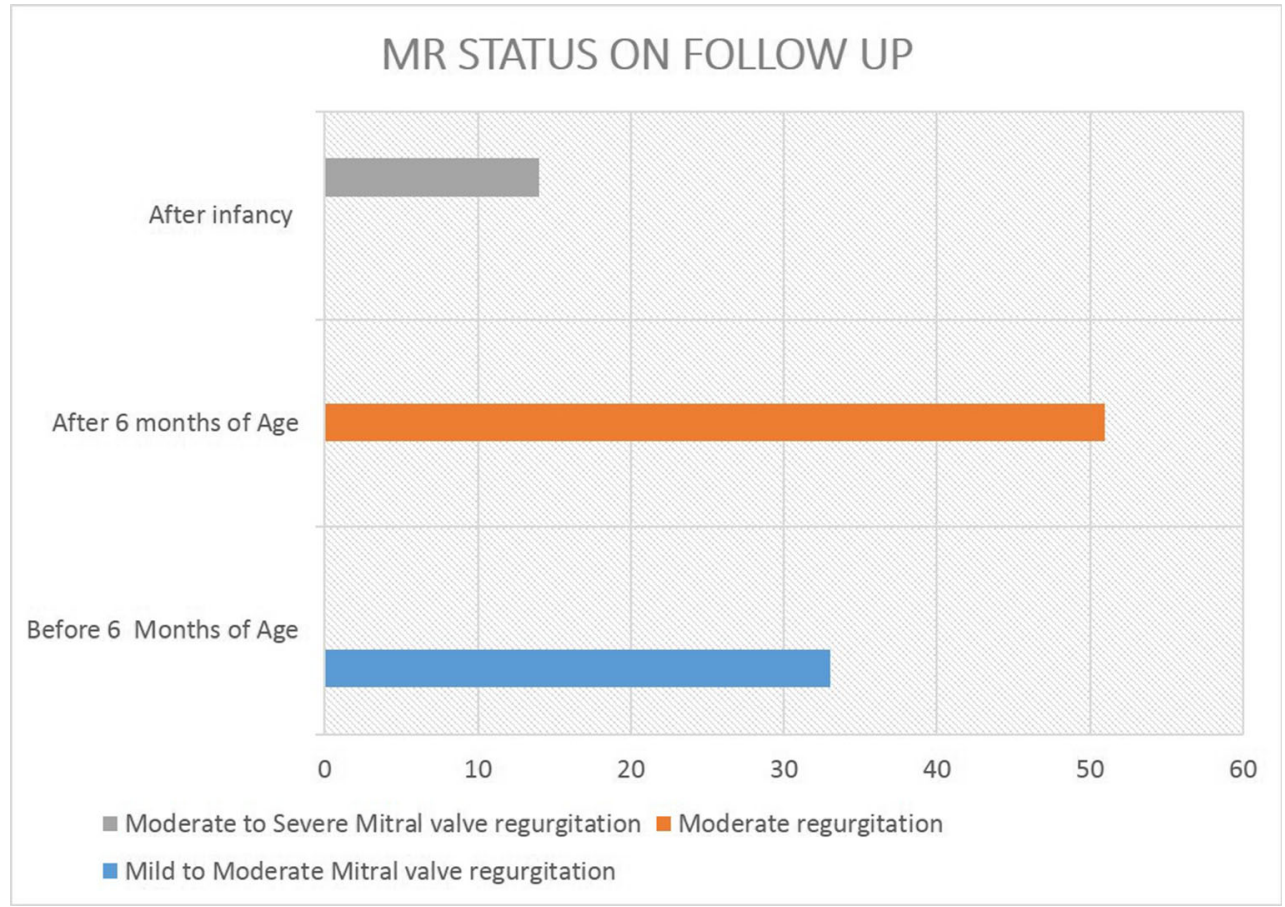


coronary and directly anastomose it to the aorta, on its postero-lateral aspect. Direct aortic implantation has been shown to have a higher rate of late patency [13]; Ben Ali et al. had demonstrated that 2 of their 61 patients required re-operation for coronary stenosis after direct aortic implantation [14]. Post-operative coronary stenosis should be suspected in a patient with persistent LV dysfunction or worsening MR, although we have not come across any such incidence in our series.

Our experience with ALCAPA in adult patients is limited, as most of these are managed by cardiac surgeons for adults, and they usually perform coronary artery bypass surgery (CABG) with ligation of proximal left circumflex (LCX) coronary artery for such patients $[15,16]$. In our two adult patients, we could mobilise the coronary and could relocate it on aorta, using the trap door technique. However, studies suggest that Takeuchi technique is a better technique for ALCAPA in adult patients, especially when the left coronary artery is arising from the non-facing sinus and in adult patients where mobilisation is difficult [17]. However, late baffle leak and pulmonary stenosis each have high incidence, necessitating lifelong care. Even baffle obstruction and aortic regurgitation have been reported [17]. We have no practical experience with Takeuchi's operation.

\section{Left ventricular function}

LV dysfunction has been reported as the main risk factor for perioperative mortality $[18,19]$. We have observed that there is the minimal improvement in LVEF on the day of surgery. However, in most of the patients operated in early infancy, improvement in LV function and ejection fraction was seen in a week or so. In the patients operated in late infancy, LV function may not improve at all, even at the time of discharge. Improvement in LV function is ultra-slow in the follow-up period of the first 3 months. Patients operated in early infancy or as newborns have better post-operative recovery and speedy recovery of their LV function on follow-up [20, 21]. Despite good clinical condition and normalised LVEF (more than 55\%) in ALCAPA patients after surgical repair in the long-term follow-up, the diastolic and longitudinal systolic function and LV torsion of all cardiac chambers remained impaired, especially in the LCA region. Lifelong surveillance is mandatory $[22,23]$, as ischemia can lead to myocardial scarring, even in early infancy. The recovery of LV function is closely related to scar burden [24]. In our experience, except for the patient who had left to right shunt, severe PAH and accessory LAD, patients operated within 1 month of birth or early infancy had the best outcomes. In contrast to the series reported by Monge et al. [25], we never came across any patient who underwent surgery in late infancy and had normal LV function during follow-up. Probably this is because of late diagnosis and delayed presentation in developing countries, like ours [18]. However, most of our patients showed gradual improvement in LV function and symptomatic improvement on after-load reducing medicines.

\section{Mitral regurgitation}

MR is an important part of the disease and, to an extent, denotes the severity of ischemic damage of the LV. However, controversy still exists over the initial management of MR, with some groups recommending MV repair at the time of ALCAPA repair [24, 25]. Various studies have suggested that the cause of MR is LV ischemia and in the majority of patients, MR improves after the surgery.

Most centres do not recommend MV repair during initial operation, owing to the fear of prolongation of the crossclamp time in the setting of an already-compromised myocardium. Medical practitioners generally believe that with the improvement of LV function and dimensions after successful revascularisation, the mitral annular size shall decrease with subsequent improvement in MR. However, our experience does not corroborate this view point, and for patients who come in late infancy and childhood with severe MR, where the cause of MR is ischemic lesions of the papillary muscle (organic MR), we recommend MV repair [18, 19]. In this group, mitral annuloplasty in association with coronary reimplantation is indicated due to the reduced likelihood of regression of MR after coronary revascularisation [26]. If the MV is not repaired in these patients during surgery, their postoperative course is difficult and most likely will need reoperation for MR. Severe MR leads to prolonged ventilation and it is extremely tough weaning from the ventilator. So, since 2012, we electively repair MV in patients with moderate to severe MR using posterior suture annuloplasty [18, 20,26]. We have observed that in the patients, who underwent MV repair for severe MR, even if there was residual mild to moderate MR, their post-operative course in ICU was smooth. Although most of the patients regain their LV function and there is improvement in the degree of MR with coronary revascularisation, few patients still show the sequel of myocardial damage and a hemodynamically significant degree of $\mathrm{MR}$, requiring close follow-up and further surgical intervention. Reduction annuloplasty, with or without chordal shortening and quadrangular resection, is the procedure of choice for the ischemic MR in children. Otherwise, MV replacement with mechanical prosthesis should be the last resort for patients with MR refractory to conventional repair [21].

For ALCARPA, we have reviewed and summarised cases reported in the literature [4]. Coronary reimplantation without unroofing and unroofing alone have their advantages and disadvantages. We found that the unroofing technique, which we have used in our patients, is simple and reproducible. When encountered, unroofing is always a preferred technique, as compared to reimplantation. The main problem of reimplantation 
technique for ALCARPA is the risk of "kinks" of the coronary artery as the left coronary button has to be rotated almost 90-180 degrees for reimplantation. The transfer site would be far above the sinus of Valsalva, almost at the mid-portion of the ascending aorta, which adversely affects LCA blood pattern, as compared to the case whose LCA has the origin inside the sinus [27]. The consistent presence of an intramural segment in the intramural coronary artery is known to pose potential risks of myocardial ischemia, and even sudden death [28]. The unroofing procedure would seem to be superior to reimplantation, because it can normalise virtually the entire course of the LCA, without leaving the intramural segment, which could eliminate a potential nidus for lethal events and it is technically simple too. We perform unroofing with the same technique that we had described for transposition of great arteries with intramural coronary artery [29]. We believe that unroofing leads to uniform coronary flow from the sinus of Valsalva.

Imaging almost always fails to diagnose intramural course. Echocardiogram fails to detect the aortic intramural course, due to a vertical course of the anomalous LCA parallel to ascending aortic wall, with the intimal relationship between the aorta and anomalous LCA. CT angiography also failed to diagnose the intramural aortic course in two of our cases. However, on reviewing the literature, indicators of latter are as follows: (1) high origin of the LCA at the pulmonary trunk or on the right PA, (2) ascending and parallel course of the ALCAPA to the aortic wall and (3) presence of a left perpendicular angle formed by the junction of the vertical intramural aortic course [30,31]. It is not that ALCARPA always has an intramural course. Rarely, the LCA may arise from right PA with the direct extravascular course and in such a situation, one has to relocate the coronary artery by turning the ostium by 180 degrees. Even single coronary artery origin from right PA, without any intramural course, has been reported in English medical literature [32, 33].

Both infants and adult patients with left main coronary artery atresia have been reported [5, 34, 35]. Adults may present as patients of ischemic heart disease and only during angiography may be diagnosed to have left main atresia. A 76year-old asymptomatic lady was diagnosed to have left main coronary atresia during the autopsy. We believe that patients with left main coronary artery atresia are the worst subset of AOCAPA and have a feeble LV function and severe MR at the time of diagnosis, with extensive collaterals. Surgical revascularisation using a bypass graft is reported for this subset of patients. However, in two of our patients, as the length of the bulbous left main coronary stump was adequate, with proximity to ascending aorta, we could transfer the left main coronary artery directly to the aorta. This technique is not reported in English medical literature to date (Fig. 7a-e).

For all our patients with ARCAPA, who were adolescent, asymptomatic and diagnosed elsewhere and referred to us, the dilemma existed regarding surgical intervention versus conservative management. Radke and colleagues [36], in a review, found that $40 \%$ of the patient were symptomatic and their study also confirmed the inability of the large dilated coronary arteries to appropriately fill the coronary sinus, owing to the steal phenomena with poor coronary flow reserve, until surgical correction. Mintz et al. [37] have also demonstrated the coronary steal phenomena in adult patients with ARCAPA. We believe that even if the patients are asymptomatic, they should be advised for surgical correction to establish two-coronary supply.

The literature search also revealed an extremely rare form of the anomalous origin of the single coronary artery from the PA, with successful surgical intervention in infancy [38]. We never came across such an anomaly in our series.

\section{Conclusion}

We believe and advocate that all cases of AOCAPA need surgical correction; otherwise, the patients will continue to have a progressively increasing left to right shunt and poor coronary flow, which will predispose to ischemia, arrhythmias and sudden death. Although echocardiography is diagnostic for most of these anomalies, there are other unusual presentations and variants, which may require definitive imaging using $\mathrm{CT}$ and magnetic resonance imaging. They are emerging as applicable diagnostic imaging modalities. One should always keep in mind the possibility of left main coronary atresia, ALCARPA and single coronary artery origin from the PA, as these are rare forms of AOCAPA. Nowadays, coronary reimplantation technique to re-establish the normal dual coronary supply has become the method of choice, with early mortality rate approaching to zero. LV dysfunction has been reported as the main risk factor for perioperative mortality. We routinely use prophylactic infusion of levosimendan in paediatric patients with impaired LV function. In patients operated in late infancy or childhood, LV function may not improve until the time of discharge, but gradual improvement in LV function and degree of MR is observed even after 7-8 years of surgery. We believe that the newborns and early infancy patients have better post-operative recovery and speedy recovery of their LV function. As the diastolic, longitudinal systolic function and LV torsion remain impaired, lifelong surveillance of ALCAPA patients is needed.

In our experience, in patients who had presented in late infancy and childhood with severe MR, the major cause of MR was ischemic lesions of the papillary muscles (organic MR). In this group, mitral annuloplasty, in association with coronary reimplantation, is indicated, due to the reduced likelihood of regression of MR after coronary revascularisation alone. The presence of left to right shunt provides accessory oxygenated blood supply to the LV and preserves the ventricular function. The presence of additional or accessory branch 
from the RCA to the LV also helps in preserving the LV function. If there is sudden unexplained ventricular dysfunction in the postoperative patient with left to right shunt, anomalous origin of coronary artery from PA should be suspected. Early diagnosis and establishment of two coronary artery system can give the best outcome in this complex congenital condition.

Acknowledgements The author gratefully acknowledges the invaluable contributions of Mr. Sanjay Patel and Ms. Himani Pandya in preparing the manuscript and Dr. Abdul Majeed, Mr. Asish Patel and Mr. Nikunj Solanki for their uncannily lifelike operative illustrations.

Funding This work was supported by the U.N. Mehta Institute of Cardiology and Research Centre itself and received no specific grant from any funding agency, commercial or not-for-profit sectors.

\section{Compliance with ethical standards}

Ethics approval and consent to participate Ethics approval was waived off being a retrospective study of a standard surgery. Informed consent was obtained from all patients.

Conflict of interest The author declares no competing interests.

Research involving human participants and/or animals This article does not contain any studies with animals performed by the author. Study was performed in accordance with the ethical standards as laid down in the 1964 Declaration of Helsinki and its later amendments or comparable ethical standards.

\section{References}

1. Yamanaka O, Hobbs RE. Coronary artery anomalies in 126,595 patients undergoing coronary arteriography. Catheter Cardiovasc Diagn. 1990;21:28-40.

2. Peña E, Nguyen ET, Merchant N, Dennie C. ALCAPA syndrome: not just a pediatric disease. Radiographics. 2009;29:553-65.

3. Yau JM, Singh R, Halpern EJ, Fischman D. Anomalous origin of the left coronary artery from the pulmonary artery in adults: a comprehensive review of 151 adult cases and a new diagnosis in a 53year-old woman. Clin Cardiol. 2011;34:204-10.

4. Agrawal V, Vaidhya N, Patel M, Mishra A, Patel D. Intramural aortic course should always be considered for anomalous origin of the left coronary artery from the right pulmonary artery. World J Pediatr Congenit Heart Surg. 2019;10:508-12.

5. Musiani A, Cernigliaro C, Sansa M, Maselli D, De Gasperis C. Left main coronary artery atresia: literature review and therapeutical considerations. Eur J Cardiothorac Surg. 1997;11:505-14.

6. Contreras AE, Leonardi C, Lazzarin O, Bagur R, Peirone A. Anomalous origin of the right coronary artery from the pulmonary artery diagnosed as an incidental finding. Congenit Heart Dis. 2012;8:E52-5.

7. Vogt PR, Tkebuchava T, Arbenz U, Von Segesser LK, Turina MI. Anomalous origin of the right coronary artery from the pulmonary artery. Thorac Cardiovasc Surg. 1994;42:125-7.

8. Kim Y-S, Lee M, Cho YH, Yang J-H, Jun T-G. An alternative surgical technique for repair of anomalous origin of the left coronary artery from the pulmonary artery. Korean J Thorac Cardiovasc Surg. 2014;47:220-4.
9. Alhadlaq A, Dhillon S, Hancock-Friesen CL, Hussain A. A hidden culprit for ventricular dysfunction in aortopulmonary window repair: anomalous origin of left coronary artery. Case report and review of literature. J Thorac Cardiovasc Surg. 2016;152:e123-6.

10. Cooley DA, Hallman GL, Bloodwell RD. Definitive surgical treatment of anomalous origin of left coronary artery from pulmonary artery: indications and results. J Thorac Cardiovasc Surg. 1966;52: 798-808.

11. Neches WH, Mathews RA, Park SC, et al. Anomalous origin of the left coronary artery from the pulmonary artery. A new method of surgical repair. Circulation. 1974;50:582-7.

12. Meyer BW, Stefanik G, Stiles QR, Lindesmith GG, Jones JC. A method of definitive surgical treatment of anomalous origin of left coronary artery. A case report. J Thorac Cardiovasc Surg. 1968;56: 104-7.

13. Lange R, Vogt M, Hörer J, et al. Long term results of repair of anomalous origin of the left coronary artery from the pulmonary artery. Ann Thorac Surg. 2007;83:1463-71.

14. Ben Ali W, Metton O, Roubertie F, et al. Anomalous origin of the left coronary artery from the pulmonary artery: late results with special attention to the mitral valve. Eur J Cardiothorac Surg. 2009;36:244-8.

15. Harky A, Noshirwani A, Karadakhy O, Ang J. Comprehensive literature review of anomalies of the coronary arteries. J Card Surg. 2019;34:1328-43.

16. Sabik JF 3rd, Lytle BW, Blackstone EH, Khan M, Houghtaling PL, Cosgrove DM. Does competitive flow reduce internal thoracic artery graft patency? Ann Thorac Surg. 2003;76:1490-6.

17. Takeuchi S, Imamura H, Katsumoto K, Hayashi I, Katohgi T, Yozu R. New surgical method for repair of anomalous left coronary artery from pulmonary artery. J Thorac Cardiovasc Surg. 1979;78:7-11.

18. Zhang W, Hu R, Zhu Y, et al. Surgical outcomes for anomalous left coronary artery from the pulmonary artery: influence of late presentation. J Thorac Cardiovasc Surg. 2020;159:1945-52.

19. Di Salvo G, Siblini G, Issa Z, et al. Left ventricular mechanics in patients with abnormal origin of the left main coronary artery from the pulmonary trunk late after successful repair. Cardiology. 2017;136:71-6.

20. Dehaki MG, Al-Diary A, Rezaei Y, Ghavidel AA, Omrani G, Givtaj N. Mid-term outcomes of surgical repair for anomalous origin of the left coronary artery from the pulmonary artery: in infants, children and adults. Ann Pediatr Card. 2017;10:137-43.

21. Karimi M, Kirshbom PM. Anomalous origins of coronary arteries from the pulmonary artery: a comprehensive review of literature and surgical options. World J Pediatr Congenit Heart Surg. 2015;6:526-40.

22. Dąbrowska-Kugacka A, Dorniak K, Meyer-Szary J, et al. Myocardial function in patients with anomalous left coronary artery from the pulmonary artery syndrome: a long-term speckle tracking echocardiographic study. PLoS One. 2019;14:e223227. https://doi.org/10.1371/journal.pone.0223227.

23. Memon MKY, Amanullah M, Atiq M. Anomalous left coronary artery from pulmonary artery: an important cause of ischemic mitral regurgitation in children. Cureus. 2019;11:e4441.

24. Bhalgat $\mathrm{P}$, Naik A, Salvi $\mathrm{P}$, et al. Cardiac magnetic resonance imaging, myocardial scar and coronary flow pattern in anomalous origin of left coronary artery from the pulmonary artery. Indian Heart J. 2018;70:303-7.

25. Mongé MC, Eltayeb O, Costello JM, Sarwark AE, Carr MR, Backer CL. Aortic implantation of anomalous origin of the left coronary artery from the pulmonary artery: long-term outcomes. Ann Thorac Surg. 2015;100:154-60.

26. Weixler VHM, Zurakowski D, Kheir J, et al. Fontan with lateral tunnel is associated with improved survival compared with extracardiac conduit. J Thorac Cardiovasc Surg. 2020;159:148091.e2. 
27. de Paulis R, Tomai F, Bertoldo F, et al. Coronary flow characteristics after a Bentall procedure with or without sinuses of Valsalva. Eur J Cardiothorac Surg. 2004;26:66-72.

28. Adachi I, Kagisaki K, Yagihara T, et al. Unroofing aortic intramural left coronary artery arising from right pulmonary artery. Ann Thorac Surg. 2008;85:675-7.

29. Mishra A, Jain A, Hinduja M, et al. Transposition of great arteries with intramural coronary artery: experience with a modified surgical technique. Braz J Cardiovasc Surg. 2016;31:15-21.

30. Marshall CD, Weigand J, Sambatakos P, et al. Repair of anomalous left coronary artery from the right pulmonary artery: a series of nine cases. World J Pediatr Congenit Heart Surg. 2015;6:382-6.

31. Atik E, Barbero-Marcial M, Tanamati C, Kajita L, Ebaid M, Jatene A. Anomalous origin of the left coronary artery from the right pulmonary artery with intramural aortic trajectory. Clinicosurgical diagnostic implications. Arq Bras Cardiol. 1999;73:181-90.

32. Vitanova K, Cleuziou J, Deutsch MA, Ackermann K, Schreiber C. A rare anomaly of a single coronary artery arising from the right pulmonary artery in a neonate. World J Pediatr Congenit Heart Surg. 2014;5:453-5.

33. Tavora F, Burke A, Kutys R, Li L, Virmani R. Total anomalous origin of the coronary circulation from the right pulmonary artery. Cardiovasc Pathol. 2008;17:246-9.
34. Sohn SY, Jang GY, Choi BM. Congenital atresia of the left main coronary artery in an infant. J Zhejiang Univ Sci B. 2010;11:53941.

35. Gebauer R, Cerny S, Vojtovic P, Tax P. Congenital atresia of the left coronary artery-myocardial revascularization in two children. Interact Cardiovasc Thorac Surg. 2008;7:1174-5.

36. Radke PW, Messmer BJ, Haager PK, Klues HG. Anomalous origin of the right coronary artery: preoperative and postoperative hemodynamics. Ann Thorac Surg. 1998;66:1444-9.

37. Mintz GS, Iskandrian AS, Bemis CE, Mundth ED, Owens JS. Myocardial ischemia in anomalous origin of the right coronary artery from the pulmonary trunk: proof of a coronary steal. Am J Cardiol. 1983;51:610-2.

38. Mirza A, Bergoend E, Legendre A, Neville P. Successful surgical treatment for a single coronary artery arising from the pulmonary artery. Pediatr Cardiol. 2008;29:1107-9.

Publisher's note Springer Nature remains neutral with regard to jurisdictional claims in published maps and institutional affiliations. 